\title{
Existence of Positive Solutions for a Fourth-Order $m$-Point Boundary Value Problem
}

\author{
Liu Yang and Chunfang Shen \\ Department of Mathematics, Hefei Normal University, Hefei, Anhui 230061, China \\ Correspondence should be addressed to Liu Yang; yliu722@163.com
}

Received 3 August 2014; Accepted 5 February 2015

Academic Editor: Jesús G. Falset

Copyright (c) 2015 L. Yang and C. Shen. This is an open access article distributed under the Creative Commons Attribution License, which permits unrestricted use, distribution, and reproduction in any medium, provided the original work is properly cited.

By using Krasnosel'skii's fixed point theorem and the fixed point index theorem in the special function space, we obtain some sufficient conditions for the existence of positive solutions of fourth-order boundary value problem with multipoint boundary conditions. Applications of our results to some special problems are also discussed.

\section{Introduction}

In this paper, we study the existence of positive solutions of the boundary value problem consisting of the nonlinear fourth-order differential equation

$$
u^{(4)}(t)+a(t) f(u(t))=0, \quad t \in[0,1]
$$

and the multipoint boundary condition

$$
\begin{gathered}
u^{\prime \prime \prime}(0)=0, \quad u^{\prime \prime}(0)=0, \quad u^{\prime}(0)=0, \\
u(1)=\sum_{i=1}^{m-2} \beta_{i} u\left(\xi_{i}\right)
\end{gathered}
$$

or

$$
\begin{gathered}
u^{\prime \prime \prime}(1)=0, \quad u^{\prime \prime}(1)=0, \quad u^{\prime}(1)=0, \\
u(0)=\sum_{i=1}^{m-2} \beta_{i} u\left(\xi_{i}\right),
\end{gathered}
$$

where $0<\xi_{1}<\xi_{2}<\cdots<\xi_{m-2}<1,0<\beta_{i}<1, i=$ $1,2, \ldots, m-2, \sum_{i=1}^{m-2} \beta_{i}<1$ and $f \in C([0,+\infty),[0,+\infty))$, $a(t) \in C([0,1],[0,+\infty))$, and there exist $t_{0} \in[0,1]$ such that $a\left(t_{0}\right)>0$.

It is well known that boundary value problems of fourthorder ordinary differential equations are used to describe a large number of physical, biological, and chemical phenomena. For an example, (1) is often used to describe the deformation of an elastic beam under a variety of boundary conditions [1-3]. For this reason, there is a wide literature that deals with the existence and the multiplicity of solutions for fourth-order boundary value problems; see [4-19].

Krasnoselskiis theorem in a cone and fixed point index theorem have often been used to study the existence and multiplicity of positive solutions of nonlocal boundary value problems over the last several years. As to fourth-order Bvps, by using Krasnoselskiis fixed point theorem, Graef et al. [11] established the existence results of positive solutions for the nonlinear fourth-order ordinary differential equation

$$
u^{(4)}(t)+\lambda g(t) f(u(t))=0, \quad t \in[0,1]
$$

with the boundary conditions

$$
u(0)=u^{\prime}(1)=u^{\prime \prime}(0)=u^{\prime \prime}(p)-u^{\prime \prime}(1)=0 .
$$

However, the aforementioned papers mainly considered the two or three point boundary conditions. There are very few works on the multipoint boundary value problem for fourth-order ordinary differential equations. As to $m$-point boundary value problem ((1), (2)) or ((1), (3)), there is no existence results of solution or positive solution. The goal of this paper is to fill this gap. In this paper, by constructing an available integral operator and combining fixed point theorem and fixed point index theorem, we establish some sufficient conditions of the existence of positive solutions for problems ((1), (2)) and ((1), (3)). 
The rest of this paper is organized as follows. In Section 2, we given some preliminaries and lemmas for use later. Section 3 is devoted to the existence and multiplicity of positive solutions of problem ((1), (2)). In Section 4, we establish the existence results of positive solutions for problem ((1), (3)). In Section 5, we give some examples to demonstrate the main results of this paper.

\section{Preliminaries}

Definition 1. Let $E$ be a real Banach space over the real numbers. A nonempty convex closed set $P \subset E$ is said to be a cone provided that

(i) $a u \in P$, for all $u \in P, a \geq 0$,

(ii) $u,-u \in P$ implies $u=0$.

Definition 2. An operator is called completely continuous if it is continuous and maps bounded sets into precompact sets.

Let $E$ be the Banach space $C[0,1]$ endowed with the norm $\|u\|=\max _{0 \leq t \leq 1}|u(t)|$. We denote

$$
f_{0}=\lim _{u \rightarrow 0^{+}} \frac{f(u)}{u}, \quad f_{\infty}=\lim _{u \rightarrow \infty} \frac{f(u)}{u} .
$$

Lemma 3 (see [20]). Let E be a Banach space and $K \subset E$ be a cone. Assume $\Omega_{1}$ and $\Omega_{2}$ are open bounded subsets of $E$ with $0 \in \Omega_{1} \subset \bar{\Omega}_{1} \subset \Omega_{2}$, and let

$$
A: K \cap\left(\bar{\Omega}_{2} \backslash \Omega_{1}\right) \longrightarrow K
$$

be a completely continuous operator such that

$$
\begin{array}{cc}
\|A u\| \leq\|u\|, \quad & u \in K \cap \partial \Omega_{1} \\
\|A u\| \geq\|u\|, & u \in K \cap \partial \Omega_{2}
\end{array}
$$

or

$$
\begin{aligned}
& \|A u\| \geq\|u\|, \quad u \in K \cap \partial \Omega_{1}, \\
& \|A u\| \leq\|u\|, \quad u \in K \cap \partial \Omega_{2} ;
\end{aligned}
$$

then, $A$ has a fixed point in $K \cap\left(\bar{\Omega}_{2} \backslash \Omega_{1}\right)$.

Lemma 4 (see [20]). Let $E$ be a Banach space and let $K \subset E$ be a cone. For $\rho>0$, define $K_{\rho}=\{u \in K:\|u\| \leq \rho\}$ and assume that $A: K_{\rho} \rightarrow K$ is a completely continuous operator such that $A u \neq u$ for $x \in \partial K_{\rho}=\{u \in K:\|u\|=\rho\}$. Then,

$$
\begin{gathered}
\|A u\| \leq\|u\|, \quad u \in \partial K_{\rho}, \text { then } i\left(A, K_{\rho}, K\right)=1, \\
\|A u\| \geq\|u\|, \quad u \in \partial K_{\rho}, \text { then } i\left(A, K_{\rho}, K\right)=0 .
\end{gathered}
$$

\section{Existence Results of Problems (1) and (2)}

We begin with the fourth-order $m$-point boundary value problem

$$
\begin{gathered}
u^{(4)}(t)+y(t)=0, \quad t \in[0,1], \\
u^{\prime \prime \prime}(0)=0, \quad u^{\prime \prime}(0)=0, \quad u^{\prime}(0)=0, \\
u(1)=\sum_{i=1}^{m-2} \beta_{i} u\left(\xi_{i}\right) .
\end{gathered}
$$

Lemma 5. Denoting $\xi_{0}=0, \xi_{m-1}=1, \beta_{0}=\beta_{m-1}=0$, and $y(t) \in C[0,1]$, problem (11), (12) has the unique solution

$$
u(t)=\int_{0}^{1} G(t, s) y(s) d s
$$

where

$$
\left\{( t , s ) \quad \left\{\begin{array}{l}
-\frac{1}{6} s^{3} \\
+\left(\frac{1}{6}-\frac{1}{2} s+\frac{1}{2} s^{2}-\frac{1}{6} \sum_{k=0}^{i-1} \beta_{k} s^{3}\right. \\
\left.+\sum_{k=i}^{m-2} \beta_{k} \xi_{k}\left(-\frac{1}{6} \xi_{k}^{2}+\frac{1}{2} \xi_{k} s-\frac{1}{2} s^{2}\right)\right) \\
-\left(1-\sum_{k=0}^{m-1} \beta_{k}\right)^{-1}, \\
+\left(\frac{1}{6} t^{3}-\frac{1}{2} s t^{2}-\frac{1}{2} s^{2} t\right. \\
\left.\quad+\sum_{k=i}^{m-2} \beta_{k} \xi_{k}\left(-\frac{1}{6} s^{2}-\frac{1}{6} \sum_{k=0}^{2} \beta_{k} s^{3} \frac{1}{2} \xi_{k} s-\frac{1}{2} s^{2}\right)\right) \\
\cdot\left(1-\sum_{k=0}^{m-1} \beta_{k}\right)^{-1},
\end{array} \leq s,\right.\right.
$$

for $\xi_{i-1} \leq s \leq \xi_{i}, i=1,2, \ldots, m-1$.

Proof. Let $G(t, s)$ be Green's function of problem $-u^{(4)}(t)=0$ with boundary condition (12). We can suppose

$$
G(t, s)=\left\{\begin{array}{c}
a_{3} t^{3}+a_{2} t^{2}+a_{1} t+a_{0}, \\
t \leq s, \quad \xi_{i-1} \leq s \leq \xi_{i}, \quad i=1,2, \ldots, m-1, \\
b_{3} t^{3}+b_{2} t^{2}+b_{1} t+b_{0}, \\
t \geq s, \quad \xi_{i-1} \leq s \leq \xi_{i}, \quad i=1,2, \ldots, m-1,
\end{array}\right.
$$


where $a_{i}, b_{i}, i=0,1,2,3$ are unknown coefficients. Considering the properties of Green's function and boundary condition (12), we have

$$
\begin{aligned}
& a_{3} s^{3}+a_{2} s^{2}+a_{1} s+a_{0}=b_{3} s^{3}+b_{2} s^{2}+b_{1} s+b_{0}, \\
& 3 a_{3} s^{2}+2 a_{2} s+a_{1}=3 b_{3} s^{2}+2 b_{2} s+b_{1} \\
& 6 a_{3} s+2 a_{2}=6 b_{3} s+2 b_{2} \\
& 6 a_{3}- 6 b_{3}=1 \\
& a_{3}=0 \\
& a_{2}=0 \\
& a_{1}=0 \\
& b_{3}+b_{2}+b_{1}+b_{0} \\
&=\sum_{k=0}^{i=1} \beta_{k}\left(a_{3} \xi_{k}^{3}+a_{2} \xi_{k}^{2}+a_{1} \xi_{k}+a_{0}\right) \\
&+\sum_{k=i}^{m-2} \beta_{k}\left(b_{3} \xi_{k}^{3}+b_{2} \xi_{k}^{2}+b_{1} \xi_{k}+b_{0}\right)
\end{aligned}
$$

A straightforward calculation shows that

$$
\begin{aligned}
& a_{3}=a_{2}=a_{1}=0 \text {, } \\
& a_{0}=-\frac{1}{6} s^{3} \\
& +\left(\frac{1}{6}-\frac{1}{2} s+\frac{1}{2} s^{2}-\frac{1}{6} \sum_{k=0}^{i-1} \beta_{k} s^{3}\right. \\
& \left.+\sum_{k=i}^{m-2} \beta_{k} \xi_{k}\left(-\frac{1}{6} \xi_{k}^{2}+\frac{1}{2} \xi_{k} s-\frac{1}{2} s^{2}\right)\right) \\
& \cdot\left(1-\sum_{k=0}^{m-1} \beta_{k}\right)^{-1} \\
& b_{3}=-\frac{1}{6}, \quad b_{2}=\frac{s}{2}, \quad b_{1}=-\frac{s^{2}}{2}, \\
& b_{0}=\left(\frac{1}{6}-\frac{1}{2} s+\frac{1}{2} s^{2}-\frac{1}{6} \sum_{k=0}^{i-1} \beta_{k} s^{3}\right. \\
& \left.+\sum_{k=i}^{m-2} \beta_{k} \xi_{k}\left(-\frac{1}{6} \xi_{k}^{2}+\frac{1}{2} \xi_{k} s-\frac{1}{2} s^{2}\right)\right) \\
& \cdot\left(1-\sum_{k=0}^{m-1} \beta_{k}\right)^{-1}
\end{aligned}
$$

These give the explicit expression of Green's function. Then, we have

$$
u(t)=\int_{0}^{1} G(t, s) y(s) d s
$$

Lemma 6. One can see that $G(t, s) \geq 0, t, s \in[0,1]$.

Proof. For $\xi_{i-1} \leq s \leq \xi_{i}, i=1,2, \ldots, m-1$,

$$
\frac{\partial G(t, s)}{\partial t}= \begin{cases}0, & t \leq s, \quad \xi_{i-1} \leq s \leq \xi_{i} \\ -\frac{1}{2}(t-s)^{2}, & t \geq s, \quad \xi_{i-1} \leq s \leq \xi_{i}\end{cases}
$$

Then, $\partial G(t, s) / \partial t \leq 0,0 \leq t, s \leq 1$, which induces that $G(t, s)$ is decreasing on $t$. By a simple computation, we see

$$
\begin{aligned}
G(1, s)= & -\frac{1}{6}+\frac{1}{2} s-\frac{1}{2} s^{2} \\
& +\left(\frac{1}{6}-\frac{1}{2} s+\frac{1}{2} s^{2}-\frac{1}{6} \sum_{k=0}^{i-1} \beta_{k} s^{3}\right. \\
& \left.+\sum_{k=i}^{m-2} \beta_{k} \xi_{k}\left(-\frac{1}{6} \xi_{k}^{2}+\frac{1}{2} \xi_{k} s-\frac{1}{2} s^{2}\right)\right) \\
& \cdot\left(1-\sum_{k=0}^{m-1} \beta_{k}\right)^{-1} \\
= & \left(\frac{1}{6} \sum_{k=0}^{m-1} \beta_{k}(1-s)^{3}+\frac{1}{6} \sum_{k=i}^{m-2} \frac{1}{2} \beta_{k}\left(s-\xi_{k}\right)^{3}\right) \\
& \cdot\left(1-\sum_{k=0}^{m-1} \beta_{k}\right)^{-1} \\
\geq & 0 .
\end{aligned}
$$

This ensures that $G(t, s) \geq 0, t, s \in[0,1]$.

Lemma 7. If $y(t) \geq 0, t \in[0,1]$ and $u(t)$ is the solution of problem (11), (12), then

$$
\min _{0 \leq t \leq 1}|u(t)| \geq \gamma \max _{0 \leq t \leq 1}|u(t)|
$$

where $\gamma=\sum_{i=1}^{m-2} \beta_{i}\left(1-\xi_{i}\right) /\left(1-\sum_{i=1}^{m-2} \beta_{i} \xi_{i}\right)$.

Proof. Since $u^{(4)}(t) \leq 0, t \in[0,1]$, then $u^{\prime \prime \prime}(t)$ is decreasing on $[0,1]$. Considering $u^{\prime \prime \prime}(0)=0$, we have $u^{\prime \prime \prime}(t) \leq 0, t \in[0,1]$. Thus, $u^{\prime \prime}(t)$ is decreasing on $[0,1]$. Considering this together with the boundary condition $u^{\prime \prime}(0)=0$, we conclude that $u^{\prime \prime}(t) \leq 0$. Then, $u(t)$ is concave on $[0,1]$. Taking into account that $u^{\prime}(0)=0$, we get that

$$
\max _{0 \leq t \leq 1} u(t)=u(0), \quad \min _{0 \leq t \leq 1} u(t)=u(1)
$$

From the concavity of $u(t)$, we have

$$
\xi_{i}(u(1)-u(0)) \leq u\left(\xi_{i}\right)-u(0) .
$$


Multiplying both sides with $\beta_{i}$ and considering the boundary condition, we have

$$
\begin{aligned}
\min _{0 \leq t \leq 1}|u(t)| & =u(1) \\
& \geq \frac{\left(\sum_{i=1}^{m-2} \beta_{i}\left(1-\xi_{i}\right)\right)}{\left(1-\sum_{i=1}^{m-2} \beta_{i} \xi_{i}\right) u(0)} \\
& =\gamma \max _{0 \leq t \leq 1}|u(t)| .
\end{aligned}
$$

Problem (1), (2) has a solution $u=u(t)$ if and only if $u$ solves the operator equation

$$
u(t)=\int_{0}^{1} G(t, s) a(s) f(u(s)) d s:=A u(t), \quad 0 \leq t \leq 1 .
$$

Denote the cone

$$
K=\left\{u \in E: u \geq 0, \min _{0 \leq t \leq 1} u(t) \geq \gamma\|u\|\right\} .
$$

Since $K$ is a cone, by Lemma 7 we have that $A(K) \subset K$. For the convenience, we denote

$$
\begin{aligned}
& M=\max _{0 \leq t \leq 1} \int_{0}^{1} G(t, s) a(s) d s, \\
& m=\min _{0 \leq t \leq 1} \int_{0}^{1} G(t, s) a(s) d s .
\end{aligned}
$$

Theorem 8. Problem (1), (2) has at least one positive solution if

$$
\text { (1) } f_{0}=0, f_{\infty}=\infty \text { or }
$$$$
\text { (2) } f_{0}=\infty, f_{\infty}=0 \text {. }
$$

Proof. Firstly, we consider case (1). By $f_{0}=0$, we choose $\varepsilon_{1}>$ 0 satisfying $\varepsilon_{1} M \leq 1$. Then, there exists $H_{1}>0$ such that

$$
f(u) \leq \varepsilon_{1} u, \quad u \leq H_{1} .
$$

Define $\Omega_{1}=\left\{u \in E:\|u\|<H_{1}\right\}$; then, for $u \in K \cap \partial \Omega_{1}$, we have

$$
\begin{aligned}
\|A u\| & =\max _{0 \leq t \leq 1} \int_{0}^{1} G(t, s) a(s) f(u(s)) d s \\
& \leq \int_{0}^{1} G(t, s) a(s) d s \times \varepsilon_{1} \times\|u\| \leq\|u\| .
\end{aligned}
$$

On the other hand, by $f_{\infty}=\infty$, choose $\varepsilon_{2}$ satisfying $\varepsilon_{2} m \gamma \geq$ 1. Then, there exists $\bar{H}_{2}>0$ such that

$$
f(u) \geq \varepsilon_{2} u, \quad \text { for } u \geq \bar{H}_{2} .
$$

Let $H_{2}=\max \left\{2 H_{1},(1 / \gamma) \bar{H}_{2}\right\}$ and let $\Omega_{2}=\{u \in E:\|u\|<$ $\left.H_{2}\right\}$. Then, for $u \in K \cap \partial \Omega_{2}$, we see $u \geq \gamma(1 / \gamma) \bar{H}_{2}=\bar{H}_{2}$. Then,

$$
\begin{aligned}
\|A u\| & =\max _{0 \leq t \leq 1} \int_{0}^{1} G(t, s) a(s) f(u(s)) d s \\
& \geq \varepsilon_{2} m u \geq \varepsilon_{2} m \gamma\|u\| \geq\|u\| .
\end{aligned}
$$

Then, by Lemma 3, $A$ has a fixed point $u$, which implies problem (1), (2) has at least one positive solution $K \cap\left(\Omega_{2} \backslash \bar{\Omega}_{1}\right)$.

Next, the case $f_{0}=\infty, f_{\infty}=0$ is considered. For $f_{0}=\infty$, there exists $\mathrm{H}_{3}>0$ such that

$$
f(u) \geq \varepsilon_{3} u, \quad \text { for } 0<u \leq H_{3},
$$

where $\varepsilon_{3}>0$ satisfies $\varepsilon_{3} m \gamma \geq 1$. Denote $\Omega_{3}=\{u \in E:\|u\|<$ $\left.H_{3}\right\}$; then, for $u \in K \cap \partial \Omega_{3}$, we have

$$
\begin{aligned}
\|A u\| & =\max _{0 \leq t \leq 1} \int_{0}^{1} G(t, s) a(s) f(u(s)) d s \\
& \geq \varepsilon_{3} m u \geq \varepsilon_{3} m \gamma\|u\| \geq\|u\| .
\end{aligned}
$$

Considering $f_{\infty}=0$, there exists $\bar{H}_{4}>0$ such that

$$
f(u) \leq \varepsilon_{4} u, \quad \text { for } u \geq \bar{H}_{4},
$$

where $\varepsilon_{4}>0$ satisfies $\varepsilon_{4} M \leq 1$. We distinguish two cases to discuss.

Case 1. Suppose that $f$ is bounded; then, there exists $N>0$ satisfying $f(u)<N$. Taking $H_{4}=\max \left\{2 H_{3}, N M_{1}\right\}$ and $\Omega_{4}=$ $\left\{u \in E:\|u\|<H_{4}\right\}$, then for $u \in K \cap \partial \Omega_{4}$, we have

$$
\begin{aligned}
\|A u\| & =\max _{0 \leq t \leq 1}|A u(t)| \\
& =\max _{0 \leq t \leq 1} \int_{0}^{1} G(t, s) a(s) f(u(s)) d s \\
& \leq N M \leq H_{4} \leq\|u\| .
\end{aligned}
$$

Case 2. Suppose that $f$ is unbounded. Since $f \in C([0,+\infty)$, $[0,+\infty))$, then there certainly exists $H_{4}>\max \left\{2 H_{3}, \overline{H_{4}}\right\}$ such that

$$
f(u) \leq f\left(H_{4}\right), \quad 0 \leq u \leq H_{4} .
$$

For $u \in K \cap \partial \Omega_{4}$, where $\Omega_{4}=\left\{u \in E:\|u\|<H_{4}\right\}$, we have

$$
\begin{aligned}
\|A u\| & =\max _{0 \leq t \leq 1} \int_{0}^{1} G(t, s) a(s) f(u(s)) d s \\
& \leq M f\left(H_{4}\right) \leq \varepsilon_{4} M H_{4} \leq\|u\| .
\end{aligned}
$$

So, in either case, we can choose $H_{4}>0$ such that $\|A u\| \leq\|u\|$, for $u \in K \cap \partial \Omega_{4}$. Then, Lemma 3 implies that $A$ has a fixed point. Consequently, problem (1), (2) has at least one positive solution.

Theorem 9. Problem (1), (2) has at least one positive solution in one of the following cases:

(3) $f_{0}=a \in(0,+\infty), f_{\infty}=b \in(0,+\infty)$, and $a M<1$, $b m \gamma>1$;

(4) $f_{0}=a \in(0,+\infty), f_{\infty}=b \in(0,+\infty)$, and $b M<1$, $a m \gamma>1$;

(5) $f_{0}=a \in(0,+\infty), f_{\infty}=\infty$, and $a M<1$;

(6) $f_{0}=a, f_{\infty}=0$, and $a m \gamma>1$; 
(7) $f_{0}=0, f_{\infty}=b \in(0,+\infty)$, and $b m \gamma>1$;

(8) $f_{0}=\infty, f_{\infty}=b$, and $b M<1$.

Proof. We consider the case $f_{0}=a \in(0,+\infty), f_{\infty}=b \in$ $(0,+\infty)$ firstly. Since $f_{0}=a \in(0,+\infty)$, for $\varepsilon_{5}>0$ satisfying $\left(a+\varepsilon_{5}\right) M \leq 1$, there exists $H_{5}>0$ such that

$$
f(u) \leq\left(a+\varepsilon_{5}\right) u, \quad \text { for } u \leq H_{5} .
$$

Define $\Omega_{5}=\left\{u \in E:\|u\|<H_{5}\right\}$; then, for $x \in K \cap \partial \Omega_{5}$, we see

$$
\begin{aligned}
\|A u\| & =\max _{0 \leq t \leq 1} \int_{0}^{1} G(t, s) a(s) f(u(s)) d s \\
& \leq\left(a+\varepsilon_{5}\right) M u \leq\|u\| .
\end{aligned}
$$

Considering $f_{\infty}=b$, there exists $\bar{H}_{6} \geq 0$ and $\varepsilon_{6}$ satisfies $(b-$ $\left.\varepsilon_{6}\right) m \gamma \geq 1$ such that

$$
f(u) \geq\left(b-\varepsilon_{6}\right) u, \quad \text { for } u \geq \bar{H}_{6} .
$$

Let $H_{6}=\max \left\{2 H_{5},(1 / \gamma) \bar{H}_{6}\right\}$ and let $\Omega_{6}=\{u \in E:\|u\|<$ $\left.H_{6}\right\}$; then, for $u \in K \cap \partial \Omega_{6}$, we get $u \geq \bar{H}_{6}$; then,

$$
\begin{aligned}
\|A u\| & =\max _{0 \leq t \leq 1} \int_{0}^{1} G(t, s) a(s) f(u(s)) d s \\
& \geq\left(b-\varepsilon_{6}\right) m u \geq\left(b-\varepsilon_{6}\right) m \gamma\|u\| \geq\|u\| .
\end{aligned}
$$

Then, by Lemma 3, problem (1), (2) has at least one positive solution. As for other cases (4), (5), (6), (7), (8), the proof is considerably analogous with the case $(1),(2),(3)$ and is omitted here.

Theorem 10. Assume following conditions are satisfied:

$$
\begin{aligned}
& \left(C_{1}\right) f_{0}=f_{\infty}=0 ; \\
& \left(C_{2}\right) \text { there exist } r^{*}>0 \text { such that } f(u) \geq r^{*} / m \text { for } u \in \\
& {\left[r^{*}, r^{*} / \gamma\right] ;}
\end{aligned}
$$

then, problem (1), (2) has at least two positive solutions $u_{1}, u_{2}$ satisfying

$$
0 \leq\left\|u_{1}\right\| \leq r^{*} \leq\left\|u_{2}\right\|
$$

Proof. Considering $f_{0}=0$, similarly with Theorem 8 , we claim there exists $r_{1} \in\left(0, r^{*}\right)$ such that

$$
i\left(A, K_{r_{1}}, K\right)=1, \quad \text { where } K_{r_{1}}=\left\{u \in E:\|u\|<r_{1}\right\} \text {. }
$$

Since $f_{\infty}=0$, similarly with above, there exists $r_{2} \in\left(r^{*},+\infty\right)$ such that

$$
i\left(A, K_{r_{2}}, K\right)=1, \quad \text { where } K_{r_{2}}=\left\{u \in E:\|u\|<r_{2}\right\} \text {. }
$$

For $u \in K \cap \partial K_{r^{*}}, K_{r^{*}}=\left\{u \in E:\|u\|<r^{*}\right\}$,

$$
\begin{aligned}
\|A u\| & =\max _{0 \leq t \leq 1} \int_{0}^{1} G(t, s) a(s) f(u(s)) d s \\
& \geq m \times \frac{r^{*}}{m}=r^{*} \geq\|u\| .
\end{aligned}
$$

Hence, $i\left(A, K_{r^{*}}, K\right)=0$.
Therefore,

$$
i\left(A, K_{r^{*}} \backslash K_{r_{1}}, K\right)=-1, \quad i\left(A, K_{r_{2}} \backslash K_{r^{*}}, K\right)=1 .
$$

Thus, there exists at least two positive solutions $u_{1}, u_{2}$ such that

$$
0<\left\|u_{1}\right\| \leq r^{*} \leq\left\|u_{2}\right\|
$$

Theorem 11. Assume the following conditions are satisfied:

$\left(C_{3}\right) f_{0}=f_{\infty}=\infty$;

$\left(C_{4}\right)$ there exists $R^{*}>0$ such that $f(u) \leq R^{*} / M$ for $u \in$ $\left[R^{*}, R^{*} / \gamma\right]$

then, problem (1), (2) has at least two positive solutions $u_{1}, u_{2}$ satisfying

$$
0 \leq\left\|u_{1}\right\| \leq R^{*} \leq\left\|u_{2}\right\|
$$

Proof. Considering $f_{0}=\infty, f_{\infty}=\infty$, we claim there exists $R_{1} \in\left(0, R^{*}\right), R_{2} \in\left(R^{*},+\infty\right)$ such that

$$
\begin{aligned}
& i\left(A, K_{R_{1}}, K\right)=0, \quad \text { where } K_{R_{1}}=\left\{u \in E:\|u\|<R_{1}\right\}, \\
& i\left(A, K_{R_{2}}, K\right)=0, \quad \text { where } K_{R_{2}}=\left\{u \in E:\|u\|<R_{2}\right\} .
\end{aligned}
$$

For $u \in K \cap \partial K_{R^{*}}, K_{R^{*}}=\left\{u \in E:\|u\|<R^{*}\right\}$,

$$
\begin{aligned}
\|A u\| & =\max _{0 \leq t \leq 1} \int_{0}^{1} G(t, s) a(s) f(u(s)) d s \\
& \leq M \times \frac{R^{*}}{M}=R^{*} \leq\|u\| .
\end{aligned}
$$

Hence, $i\left(A, K_{R^{*}}, K\right)=1$. Therefore,

$$
i\left(A, K_{R^{*}} \backslash K_{R_{1}}, K\right)=1, \quad i\left(A, K_{R_{2}} \backslash K_{R^{*}}, K\right)=-1 .
$$

Thus, there exists at least two positive solutions $u_{1}, u_{2}$ such that

$$
0<\left\|u_{1}\right\| \leq R^{*} \leq\left\|u_{2}\right\|
$$

\section{Existence Results of Problem (1), (3)}

Lemma 12. Denoting $\xi_{0}=0, \xi_{m-1}=1$, and $\beta_{0}=\beta_{m-1}=0$, Green's function of problem

$$
\begin{gathered}
-u^{(4)}(t)=0, \\
u^{\prime \prime \prime}(1)=0, \quad u^{\prime \prime}(1)=0, \quad u^{\prime}(1)=0, \\
u(0)=\sum_{i=1}^{m-2} \beta_{i} u\left(\xi_{i}\right)
\end{gathered}
$$


is

$$
H(t, s)=\left\{\begin{array}{c}
\frac{1}{6} t^{3}-\frac{1}{2} s t^{2}+\frac{1}{2} s^{2} t \\
+\left(\sum_{k=0}^{i-1} \beta_{k}\left(\frac{1}{6} \xi_{k}^{3}-\frac{1}{2} \xi_{k}^{2} s+\frac{1}{2} \xi_{k} s^{2}\right)\right. \\
\left.+\frac{1}{6} \sum_{k=i}^{m-2} \beta_{k} s^{3}\right) \cdot\left(1-\sum_{k=0}^{m-1} \beta_{k}\right)^{-1} \\
t \leq s, \quad \xi_{i-1} \leq s \leq \xi_{i} \\
\frac{1}{6} s^{3}+\left(\sum_{k=0}^{i-1} \beta_{k}\left(\frac{1}{6} \xi_{k}^{3}-\frac{1}{2} \xi_{k}^{2} s+\frac{1}{2} \xi_{k} s^{2}\right)\right. \\
\left.+\frac{1}{6} \sum_{k=i}^{m-2} \beta_{k} s^{3}\right) \cdot\left(1-\sum_{k=0}^{m-1} \beta_{k}\right)^{-1} \\
t \geq s, \quad \xi_{i-1} \leq s \leq \xi_{i},
\end{array}\right.
$$

for $i=1,2, \ldots, m-1$.

Proof. For $\xi_{i-1} \leq s \leq \xi_{i}, i=1,2, \ldots, m-1$, we suppose

$$
H(t, s)= \begin{cases}a_{3} t^{3}+a_{2} t^{2}+a_{1} t+a_{0}, & t \leq s, \\ b_{3} t^{3}+b_{2} t^{2}+b_{1} t+b_{0}, & t \geq s .\end{cases}
$$

Considering the properties of Green's function together with the boundary condition (54), we have

$$
\begin{gathered}
a_{3} s^{3}+a_{2} s^{2}+a_{1} s+a_{0}=b_{3} s^{3}+b_{2} s^{2}+b_{1} s+b_{0}, \\
3 a_{3} s^{2}+2 a_{2} s+a_{1}=3 b_{3} s^{2}+2 b_{2} s+b_{1}, \\
6 a_{3} s+2 a_{2}=6 b_{3} s+2 b_{2}, \\
6 a_{3}-6 b_{3}=1, \quad b_{3}=0, \quad 6 b_{3}+2 b_{2}=0, \\
3 b_{3}+2 b_{2}+b_{1}=0, \\
a_{0}=\sum_{k=0}^{i=1} \beta_{k}\left(a_{3} \xi_{k}^{3}+a_{2} \xi_{k}^{2}+a_{1} \xi_{k}+a_{0}\right) \\
+\sum_{k=i}^{m-2} \beta_{k}\left(b_{3} \xi_{k}^{3}+b_{2} \xi_{k}^{2}+b_{1} \xi_{k}+b_{0}\right) .
\end{gathered}
$$

Hence,

$$
\begin{array}{r}
a_{3}=\frac{1}{6}, \quad a_{2}=-\frac{1}{2} s, \quad a_{1}=\frac{1}{2} s^{2}, \\
a_{0}=\left(\sum_{k=0}^{i-1} \beta_{k}\left(\frac{1}{6} \xi_{k}^{3}-\frac{1}{2} \xi_{k}^{2} s+\frac{1}{2} \xi_{k} s^{2}\right)\right. \\
\left.+\frac{1}{6} \sum_{k=i}^{m-2} \beta_{k} s^{3}\right) \cdot\left(1-\sum_{k=0}^{m-1} \beta_{k}\right)^{-1},
\end{array}
$$

$$
\begin{gathered}
b_{3}=0, \quad b_{2}=0, \quad b_{1}=0 \\
b_{0}=\frac{1}{6} s^{3}+\left(\sum_{k=0}^{i-1} \beta_{k}\left(\frac{1}{6} \xi_{k}^{3}-\frac{1}{2} \xi_{k}^{2} s+\frac{1}{2} \xi_{k} s^{2}\right)\right. \\
\left.+\frac{1}{6} \sum_{k=i}^{m-2} \beta_{k} s^{3}\right) \cdot\left(1-\sum_{k=0}^{m-1} \beta_{k}\right)^{-1} .
\end{gathered}
$$

The proof of Lemma 12 is completed.

Lemma 13. One can see that $H(t, s) \geq 0, t, s \in[0,1]$.

Proof. For $\xi_{i-1} \leq s \leq \xi_{i}, i=1,2, \ldots, m-1$,

$$
\frac{\partial H(t, s)}{\partial t}= \begin{cases}\frac{1}{2}(s-t)^{2}, & t \leq s, \xi_{i-1} \leq s \leq \xi_{i} \\ 0, & t \geq s, \xi_{i-1} \leq s \leq \xi_{i}\end{cases}
$$

Then, $\partial H(t, s) / \partial t \geq 0,0 \leq t, s \leq 1$, which implies that $H(t, s)$ is increasing on $t$. The fact that

$$
\begin{aligned}
H(0, s)= & \left(\sum_{k=0}^{i-1} \beta_{k}\left(\frac{1}{6} \xi_{k}^{3}-\frac{1}{2} \xi_{k}^{2} s+\frac{1}{2} \xi_{k} s^{2}\right)\right. \\
& \left.+\frac{1}{6} \sum_{k=i}^{m-2} \beta_{k} s^{3}\right) \cdot\left(1-\sum_{k=0}^{m-1} \beta_{k}\right)^{-1} \geq 0
\end{aligned}
$$

ensures that $H(t, s) \geq 0, t, s \in[0,1]$.

Lemma 14. If $y(t) \geq 0, t \in[0,1]$, and $u(t)$ is the solution of problem (53), (54), then

$$
\min _{0 \leq t \leq 1}|u(t)| \geq \gamma_{1} \max _{0 \leq t \leq 1}|u(t)|,
$$

where $\gamma_{1}=\sum_{i=1}^{m-2} \beta_{i} \xi_{i} /\left(1-\sum_{i=1}^{m-2} \beta_{i}\left(1-\xi_{i}\right)\right)$.

Proof. It follows from the same methods as Lemma 7 that $u(t)$ is concave on $[0,1]$. Taking into account that $u^{\prime}(1)=0$, one see that $u(t)$ is increasing on $[0,1]$ and

$$
\max _{0 \leq t \leq 1} u(t)=u(1), \quad \min _{0 \leq t \leq 1} u(t)=u(0) .
$$

From the concavity of $u(t)$, we have

$$
\xi_{i}(u(1)-u(0)) \leq u\left(\xi_{i}\right)-u(0) .
$$

Multiplying both sides with $\beta_{i}$ and considering the boundary condition, we have

$$
\sum_{i=1}^{m-2} \beta_{i} \xi_{i} u(1) \leq\left(1-\sum_{i=1}^{m-2} \beta_{i}\left(1-\xi_{i}\right)\right) u(0) .
$$

Thus,

$$
\min _{0 \leq t \leq 1}|u(t)| \geq \gamma_{1} \max _{0 \leq t \leq 1}|u(t)|
$$


Problem (1), (3) has a solution $u=u(t)$ if and only if $u$ solves the operator equation

$$
u(t)=\int_{0}^{1} H(t, s) a(s) f(u) d s:=A_{1} u(t), \quad 0 \leq t \leq 1 .
$$

Denote the cone

$$
K_{1}=\left\{u \in E: u \geq 0, \min _{0 \leq t \leq 1} u(t) \geq \gamma_{1}\|u\|\right\} .
$$

It is obvious that $A_{1}\left(K_{1}\right) \subset K_{1}$. We denote

$$
\begin{aligned}
& M_{1}=\max _{0 \leq t \leq 1} \int_{0}^{1} H(t, s) a(s) d s, \\
& m_{1}=\min _{0 \leq t \leq 1} \int_{0}^{1} H(t, s) a(s) d s .
\end{aligned}
$$

Theorem 15. Problem (1), (3) has at least one positive solution if

(1) $f_{0}=0, f_{\infty}=\infty$,

(2) $f_{0}=\infty, f_{\infty}=0$.

Theorem 16. Problem (1), (3) has at least one positive solution in one of the following cases:

(3) $f_{0}=a \in(0,+\infty), f_{\infty}=b \in(0,+\infty)$, and $a M_{1}<1$, $b m_{1} \gamma_{1}>1$;

(4) $f_{0}=a \in(0,+\infty), f_{\infty}=b \in(0,+\infty)$, and $b M_{1}<1$, $a m_{1} \gamma_{1}>1$;

(5) $f_{0}=a \in(0,+\infty), f_{\infty}=\infty$, and $a M_{1}<1$;

(6) $f_{0}=a, f_{\infty}=0$, and $a m_{1} \gamma_{1}>1$;

(7) $f_{0}=0, f_{\infty}=b \in(0,+\infty)$, and $b m_{1} \gamma_{1}>1$;

(8) $f_{0}=\infty, f_{\infty}=b$, and $b M_{1}<1$.

Theorem 17. Assume following conditions are satisfied:

$\left(C_{5}\right) f_{0}=f_{\infty}=0$;

$\left(C_{6}\right)$ there exist $r^{*}>0$ such that $f(u) \geq r^{*} / m_{1}$ for $u \in$ $\left[r^{*}, r^{*} / \gamma_{1}\right]$

then, problem (1), (3) has at least two positive solutions $u_{1}, u_{2}$ satisfying

$$
0 \leq\left\|u_{1}\right\| \leq r^{*} \leq\left\|u_{2}\right\|
$$

Theorem 18. Assume following conditions are satisfied:

$\left(C_{7}\right) f_{0}=f_{\infty}=\infty$;

$\left(C_{8}\right)$ there exists $R^{*}>0$ such that $f(u) \leq R^{*} / M_{1}$ for $u \in$ $\left[R^{*}, R^{*} / \gamma_{1}\right]$

then, problem (1), (3) has at least two positive solutions $u_{1}, u_{2}$ satisfying

$$
0 \leq\left\|u_{1}\right\| \leq R^{*} \leq\left\|u_{2}\right\|
$$

\section{Examples}

In this section, we given some examples to illustrate the main results established in this paper.

Example 1. Consider the fourth-order boundary value problem

$$
\begin{gathered}
u^{(4)}(t)+\pi(1-t)^{2} u^{\alpha}(t)=0, \quad 0 \leq t \leq 1, \\
u^{\prime \prime \prime}(0)=u^{\prime \prime}(0)=u^{\prime}(0)=0, \quad u(1)=\frac{1}{2} u\left(\frac{2}{3}\right),
\end{gathered}
$$

where $\alpha \in(0,1) \cup(1, \infty)$. Then, problems (71) have at least one positive solution. In fact, when $\alpha \in(0,1), f_{0}=\infty$ and $f_{\infty}=$ 0 ; when $\alpha \in(1, \infty), f_{0}=0$ and $f_{0}=\infty$. From Theorem 8 , there exists at least one positive solution of problem (71).

Example 2. Consider the fourth-order boundary value problem

$$
\begin{gathered}
u^{(4)}+(1-t)^{1 / 2}\left(u^{1 / 2}+u^{5 / 3}\right)=0, \quad 0 \leq t \leq 1, \\
u^{\prime \prime \prime}(1)=u^{\prime \prime}(1)=u^{\prime}(1)=0, \quad u(0)=\frac{2}{3} u\left(\frac{1}{2}\right) .
\end{gathered}
$$

We see that

$H(t, s)$

$$
\begin{aligned}
& \left(\frac{1}{6} t^{3}-\frac{1}{2} s t^{2}+\frac{1}{2} s^{2} t+\frac{1}{3} s^{3}, \quad t \leq s, 0 \leq s \leq \frac{2}{3},\right. \\
& =\left\{\begin{array}{l}
\frac{1}{2} s^{3}, \\
1 \geq s, 0 \leq s \leq \frac{2}{3},
\end{array}\right. \\
& =\left\{\begin{array}{l}
\frac{1}{6} t^{3}-\frac{1}{2} s t^{2}+\frac{1}{2} s^{2} t+\frac{1}{2} s^{2}-\frac{1}{4} s+\frac{1}{24}, \quad t \leq s, \frac{2}{3} \leq s \leq 1,
\end{array}\right. \\
& \frac{1}{6} s^{3}+\frac{1}{2} s^{2}-\frac{1}{4} s+\frac{1}{24}, \quad t \geq s, \frac{2}{3} \leq s \leq 1, \\
& \gamma_{1}=\frac{1}{2}, \quad M=\max _{0 \leq t \leq 1} \int_{0}^{1} H(t, s) a(s) d s=\frac{37}{1920}, \\
& m=\min _{0 \leq t \leq 1} \int_{0}^{1} H(t, s) a(s) d s=\frac{31}{3840} .
\end{aligned}
$$

It is easy to check that $f_{0}=f_{\infty}=\infty$. Taking $R=8$, we see

$$
f(u) \leq \frac{15360}{37}, \quad u \in[8,16]
$$

From Theorem 18, there exist at least two positive solutions of problem (72) such that

$$
\left\|u_{1}\right\| \leq 8 \leq\left\|u_{2}\right\|
$$

\section{Conflict of Interests}

The authors declare that there is no conflict of interests regarding the publication of this paper. 


\section{Acknowledgments}

The work is sponsored by the NSFC (11201109), Anhui Provincial Natural Science Foundation (1408085QA07), and the Higher School Natural Science Project of Anhui Province (KJ2014A200).

\section{References}

[1] A. R. Aftabizadeh, "Existence and uniqueness theorems for fourth-order boundary value problems," Journal of Mathematical Analysis and Applications, vol. 116, no. 2, pp. 415-426, 1986.

[2] C. P. Gupta, "Existence and uniqueness theorems for the bending of an elastic beam equation," Applicable Analysis, vol. 26, no. 4, pp. 289-304, 1988.

[3] M. A. del Pino and R. F. Manásevich, "Existence for a fourthorder boundary value problem under a two-parameter nonresonance condition," Proceedings of the American Mathematical Society, vol. 112, no. 1, pp. 81-86, 1991.

[4] G. Bonanno and B. Di Bella, "A boundary value problem for fourth-order elastic beam equations," Journal of Mathematical Analysis and Applications, vol. 343, no. 2, pp. 1166-1176, 2008.

[5] Z. Wei, "A class of fourth order singular boundary value problems," Applied Mathematics and Computation, vol. 153, no. 3, pp. 865-884, 2004.

[6] X. Zhang and L. Liu, "A necessary and sufficient condition of positive solutions for nonlinear singular differential systems with four-point boundary conditions," Applied Mathematics and Computation, vol. 215, no. 10, pp. 3501-3508, 2010.

[7] H. Feng, D. Ji, and W. Ge, "Existence and uniqueness of solutions for a fourth-order boundary value problem," Nonlinear Analysis: Theory, Methods \& Applications, vol. 70, no. 10, pp. 3561-3566, 2009.

[8] H. Pang and W. Ge, "Existence results for some fourthorder multi-point boundary value problem," Mathematical and Computer Modelling, vol. 49, no. 7-8, pp. 1319-1325, 2009.

[9] X. Zhang and L. Liu, "A necessary and sufficient condition for positive solutions for fourth-order multi-point boundary value problems with $p$-Laplacian," Nonlinear Analysis: Theory, Methods \& Applications, vol. 68, no. 10, pp. 3127-3137, 2008.

[10] E. Alves, T. F. Ma, and M. L. Pelicer, "Monotone positive solutions for a fourth order equation with nonlinear boundary conditions," Nonlinear Analysis. Theory, Methods \& Applications, vol. 71, no. 9, pp. 3834-3841, 2009.

[11] J. R. Graef, C. Qian, and B. Yang, "A three point boundary value problem for nonlinear fourth order differential equations," Journal of Mathematical Analysis and Applications, vol. 287, no. 1, pp. 217-233, 2003.

[12] X. Zhang and L. Liu, "Eigenvalue of fourth-order $m$-point boundary value problem with derivatives," Computers \& Mathematics with Applications, vol. 56, no. 1, pp. 172-185, 2008.

[13] J. Yang and Z. Wei, "Existence of positive solutions for fourth-order m-point boundary value problems with a onedimensional p-Laplacian operator," Nonlinear Analysis: Theory, Methods \& Applications, vol. 71, no. 7-8, pp. 2985-2996, 2009.

[14] R. Ma, "Existence of positive solutions of a fourth-order boundary value problem," Applied Mathematics and Computation, vol. 168, no. 2, pp. 1219-1231, 2005.

[15] R. Ma and J. Xu, "Bifurcation from interval and positive solutions of a nonlinear fourth-order boundary value problem," Nonlinear Analysis: Theory, Methods \& Applications, vol. 72, no. 1, pp. 113-122, 2010.
[16] C. Bai, D. Yang, and H. Zhu, "Existence of solutions for fourth order differential equation with four-point boundary conditions," Applied Mathematics Letters, vol. 20, no. 11, pp. 11311136, 2007.

[17] Y.-R. Yang, "Triple positive solutions of a class of fourth-order two-point boundary value problems," Applied Mathematics Letters, vol. 23, no. 4, pp. 366-370, 2010.

[18] E. R. Kaufmann and N. Kosmatov, "Elastic beam problem with higher order derivatives," Nonlinear Analysis. Real World Applications, vol. 8, no. 3, pp. 811-821, 2007.

[19] C. Bai, "Triple positive solutions of three-point boundary value problems for fourth-order differential equations," Computers \& Mathematics with Applications, vol. 56, no. 5, pp. 1364-1371, 2008 .

[20] D. Guo and V. Lakshmikantham, Nonlinear Problems in Abstract Cones, Academic Press, Orlando, Fla, USA, 1988. 


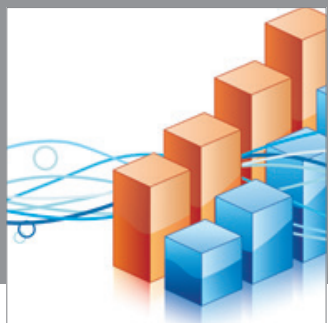

Advances in

Operations Research

mansans

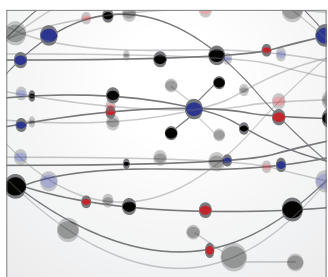

The Scientific World Journal
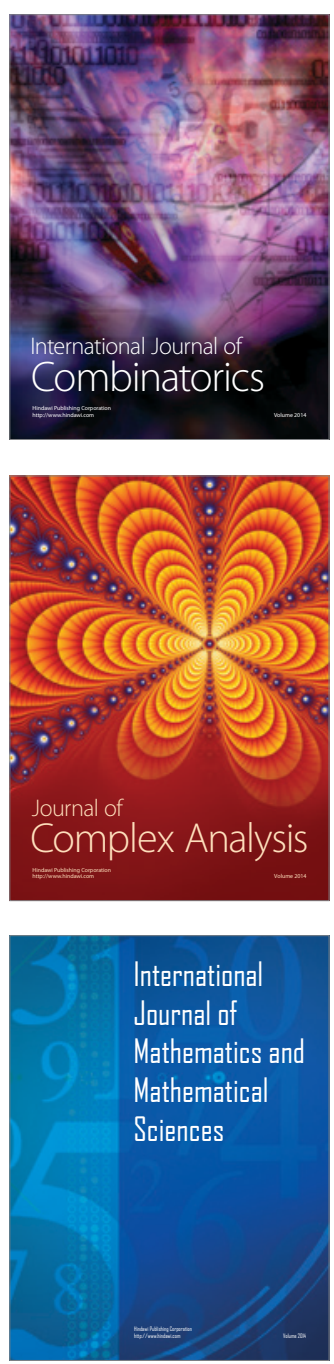
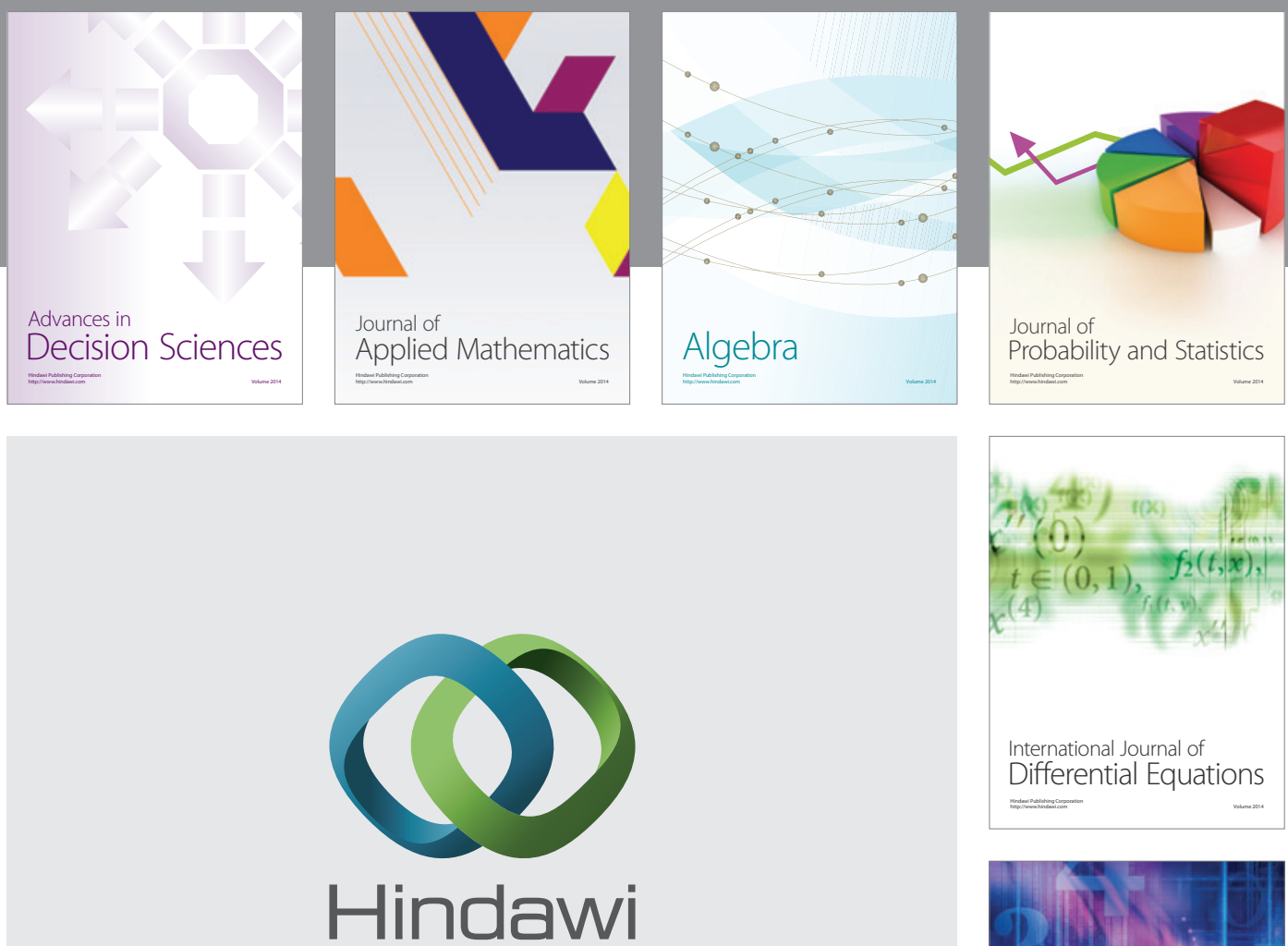

Submit your manuscripts at http://www.hindawi.com
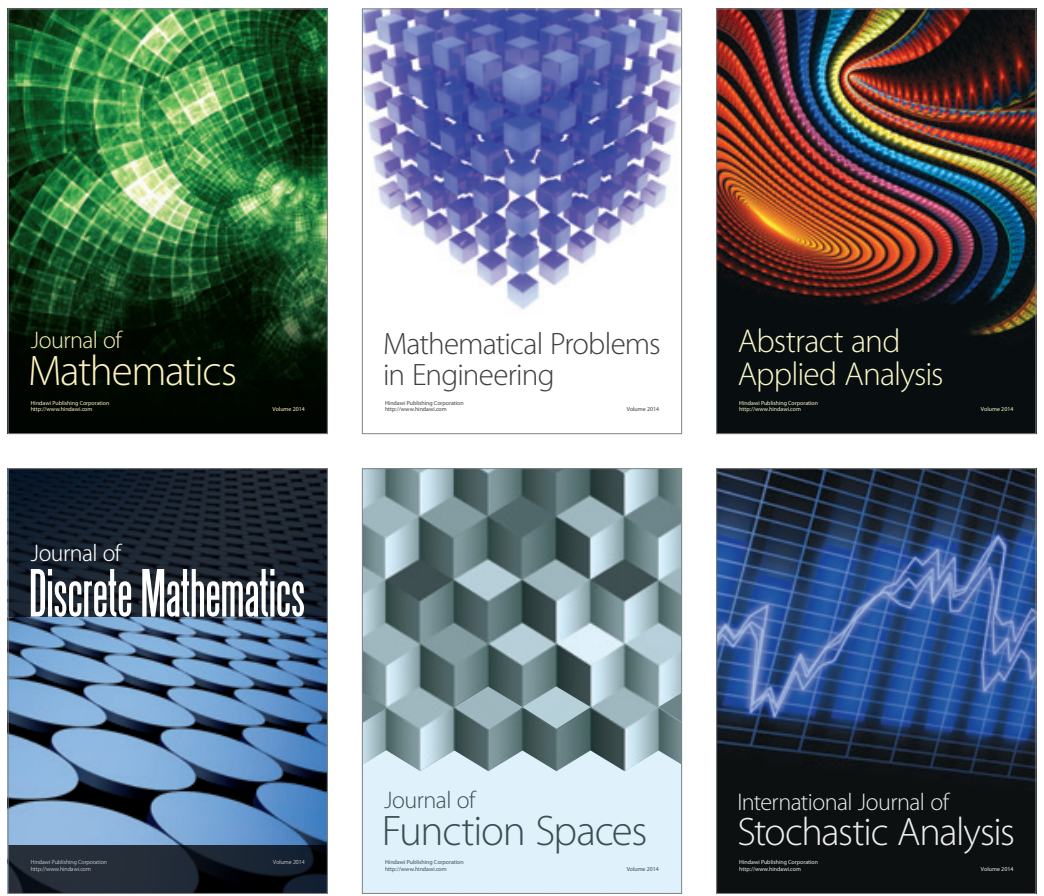

Journal of

Function Spaces

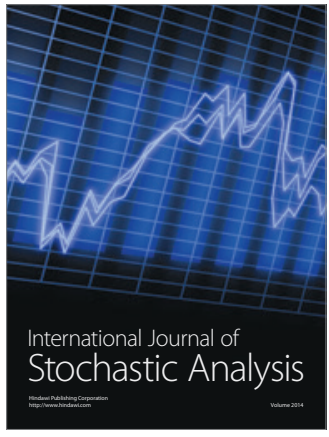

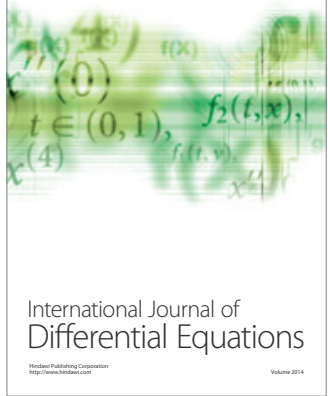
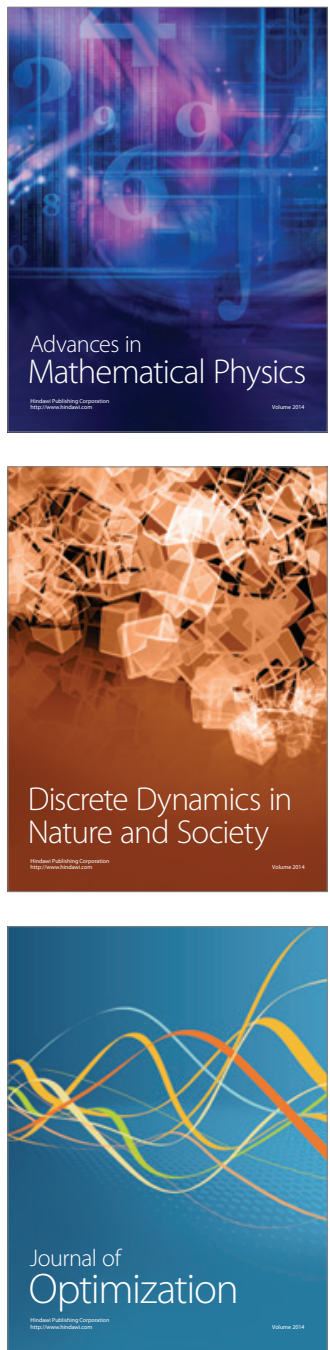